\title{
On the existence of steady-state solutions to the Navier-Stokes system for large fluxes
}

\author{
Antonio Russo And Giulio Starita
}

\begin{abstract}
In this paper we deal with the stationary Navier-Stokes problem in a domain $\Omega$ with compact Lipschitz boundary $\partial \Omega$ and datum $\boldsymbol{a}$ in Lebesgue spaces. We prove existence of a solution for arbitrary values of the fluxes through the connected components of $\partial \Omega$, with possible countable exceptional set, provided $\boldsymbol{a}$ is the sum of the gradient of a harmonic function and a sufficiently small field, with zero total flux for $\Omega$ bounded.
\end{abstract}

Mathematics Subject Classification (2000): 76D05 (primary); 31B10, 35Q30, 42B20 (secondary).

\section{Introduction}

The boundary value problem associated with the Navier-Stokes equations is to find a solution to the system

$$
\begin{aligned}
v \Delta \boldsymbol{u}-\boldsymbol{u} \cdot \nabla \boldsymbol{u} & =\nabla p & & \text { in } \Omega, \\
\operatorname{div} \boldsymbol{u} & =0 & & \text { in } \Omega, \\
\boldsymbol{u} & =\boldsymbol{a} & & \text { on } \partial \Omega,
\end{aligned}
$$

where $\Omega$ is a bounded domain (open connected set) of $\mathbb{R}^{n}(n=2,3), \boldsymbol{u}, p$ the unknown kinetic and pressure fields, $v$ the kinematical viscosity and $\boldsymbol{a}$ the boundary datum which must satisfy the condition

$$
\int_{\partial \Omega} \boldsymbol{a} \cdot \boldsymbol{n}=0
$$

where $\boldsymbol{n}$ is the outward unit normal to $\partial \Omega$ (see [6]). Existence of a variational solution

$$
(\boldsymbol{u}, p) \in\left[W_{\sigma}^{1,2}(\Omega) \cap C^{\infty}(\Omega)\right] \times\left[L^{2}(\Omega) \cap C^{\infty}(\Omega)\right]
$$

Received January 29, 2007; accepted in revised form December 13, 2007. 
to system (1.1)-(1.3) is known under the hypothesis of smallness of the fluxes

$$
\Phi_{i}=\int_{\partial \Omega_{i}} \boldsymbol{a} \cdot \boldsymbol{n}
$$

through the connected components $\partial \Omega_{i}$ of $\partial \Omega$, provided $\boldsymbol{a} \in W^{1 / 2,2}(\partial \Omega)$ and $\partial \Omega$ is Lipschitz. Precisely, in [1,5] (see also [6, Chapter VIII]) it is proved that there is a positive constant depending on $\Omega$ such that if $\sum\left|\Phi_{i}\right|$ is suitably small, then (1.1)-(1.3) has a variational solution. These results have been extended for $\boldsymbol{a}$ in Lebesgue's spaces in [10]. In particular, it is proved that if $\boldsymbol{a} \in L^{q}(\partial \Omega)(q \geq 8 / 3$ for $n=3$ and $q=2$ for $n=2$ ), then system (1.1)-(1.3) has a $C^{\infty}$ solution which for $q>4$ takes the boundary datum in the sense of nontangential convergence. Moreover, making use of some regularity results in $[2,12]$, it is showed that if $\boldsymbol{a}$ is more regular (say Hölder continuous, with Hölder's coefficient depending on the Lipschitz character of $\partial \Omega$ ) then so does $\boldsymbol{u}$.

In [3] H. Fujita and H. Morimoto considered problem (1.1)-(1.3) in a regular domain with

$$
\boldsymbol{a}=\mu \boldsymbol{u}_{0 \mid \partial \Omega}+\boldsymbol{\gamma}
$$

$\mu \in \mathbb{R}, \boldsymbol{u}_{0}=\nabla \beta$ and $\beta$ harmonic function. They proved that if $\Omega$ is regular, $\beta_{\mid \partial \Omega} \in W^{2,2}(\partial \Omega)$,

$$
\int_{\partial \Omega} \boldsymbol{\gamma} \cdot \boldsymbol{n}=0
$$

and $\|\boldsymbol{\gamma}\|_{W^{1 / 2,2(\partial \Omega)}}$ is less than a suitable constant depending on $\nu, \mu, \Omega$ and $\boldsymbol{u}_{0}$, then system (1.1)-(1.3), (1.5) admits a solution (1.4) for any $\mu \in \mathbb{R} \backslash G$, with $G$ countable subset of $\mathbb{R}$. Moreover, if $\beta \in W^{3,2}(\Omega)$ and $\|\boldsymbol{\gamma}\|_{W^{3 / 2,2}(\partial \Omega)}$ is sufficiently small, then $(\boldsymbol{u}, p) \in W_{\sigma}^{2,2}(\Omega) \times W^{1,2}(\Omega)$. This result is remarkable in view of the fact that, even though for special boundary data, it assures the existence of a solution to system (1.1)-(1.3) in arbitrary bounded regular domains for large fluxes. It is worth to mention that for the annulus $\left\{x \in \mathbb{R}^{2}: R_{1}<|x|<R_{2}\right\}$ and $\beta=\nabla \log |x|, \mathrm{H}$. Morimoto proved that $G=\varnothing[8]$ (see also [4,9]).

The aim of the present paper is twofold:

(i) to extend the results of [3] under more general assumptions on the domains and on the data and for any $n \geq 2$; in particular, for $n=3$ we show that, if $\Omega$ is a bounded Lipschitz domain, $\boldsymbol{a}$ is given by (1.5) with $\boldsymbol{u}_{0} \in W^{1, q}(\Omega), q>3 / 2$, $\gamma \in L^{2}(\partial \Omega)$ satisfies (1.6) and $\|\boldsymbol{\gamma}\|_{L^{2}(\partial \Omega)}$ is sufficiently small, then system (1.1)-(1.3) has a solution $(\boldsymbol{u}, p) \in\left[W_{\sigma}^{1 / 2,2}(\Omega) \cap C^{\infty}(\Omega)\right] \times C^{\infty}(\Omega)$ for any $\mu \in \mathbb{R} \backslash G$, with $G$ countable subset of $\mathbb{R}$;

(ii) to prove existence of a solution for system (1.1)-(1.3), (1.5) in a Lispschitz exterior domain in the class $\left[L_{\sigma}^{\infty}(\Omega, r) \cap C^{\infty}(\Omega)\right] \times\left[L^{\infty}\left(\complement S_{R}, r^{2} \log r\right) \cap C^{\infty}(\Omega)\right]$, provided $\boldsymbol{u}_{0} \in L^{\infty}\left(\Omega, r^{2}\right)$ and $\|\boldsymbol{\gamma}\|_{L^{\infty}(\partial \Omega)}$ is sufficiently small.

NotATION - We use a standard vector notation, as in [6]. Let $\Omega$ be a domain of $\mathbb{R}^{n}$, $n \geq 2$, and let $\{\gamma(\xi)\}_{\xi \in \partial \Omega}$ be a family of circular finite (not empty) cones with vertex 
at $\xi$ such that $\gamma(\xi) \backslash\{\xi\} \subset \Omega$ (as well-known, if $\Omega$ is Lipschitz, such a family of cones certainly exists). Let $\chi$ be a function in $\Omega ; \chi(x)$ is said to converge nontangentially at the boundary if $\chi(\xi)=\lim _{x \rightarrow \xi(x \in \gamma(\xi))} \chi(x) \Leftrightarrow \chi(x) \stackrel{\mathrm{nt}}{\longrightarrow} \chi(\xi)$, for almost all $\xi \in \partial \Omega$. As customary, $L^{q}(\Omega), W^{s, q}(\Omega)$ and $L^{q}(\partial \Omega), W^{s, q}(\partial \Omega)(q \in[1,+\infty], s \geq 0)$ denote respectively the Lebesgue and the Sobolev-Besov spaces of (scalar, vector and tensor) fields in $\Omega$ and $\partial \Omega$ endowed with their natural norms; $W^{-s, q}(\partial \Omega)$ is the dual space of $W^{s, q^{\prime}}(\partial \Omega)$ and $L^{\infty}(\Omega, f(r))$, with $f(r)$ positive function of $r=|x|$, is the Banach space of all measurable fields $\chi$ in $\Omega$ such that $\|f(r) \chi\|_{L^{\infty}(\Omega)}<+\infty$; if $V\left(\subset L_{\text {loc }}^{1}(\Omega)\right)$ is a function space, $V_{\sigma}$ stands for the subspace of $V$ of all (weakly) divergence free vector fields; also, the subscript $\phi$ in the symbol $W_{\phi}$, where $W \subset W^{s, q}(\partial \Omega), s \in \mathbb{R}$, denotes the set of all fields $\boldsymbol{a} \in W$ such that $\int_{\partial \Omega} \boldsymbol{a} \cdot \boldsymbol{n} \mathrm{d} \sigma=0$ or $\langle\boldsymbol{a}, \boldsymbol{n}\rangle=0$ respectively for $s \geq 0$ or $s<0$, where $\langle\cdot, \cdot\rangle$ stands for the duality pairing between $W^{s, q}(\partial \Omega)$ and its dual.

\section{Some results for the Stokes system}

The boundary-value problem associated with the Stokes system is to find a solution to the problem

$$
\begin{array}{rlrl}
v \Delta \boldsymbol{u} & =\nabla p & & \text { in } \Omega, \\
\operatorname{div} \boldsymbol{u}=0 & & \text { in } \Omega, \\
\boldsymbol{u}=\boldsymbol{a} & & \text { on } \partial \Omega .
\end{array}
$$

If $\Omega$ is exterior we require that $\boldsymbol{u}$ tends to zero at infinity.

The following theorems are proved in $[2,7,10,12]$.

Theorem 2.1. Let $\Omega$ be a Lipschitz bounded domain of $\mathbb{R}^{n}$ and let $\boldsymbol{a} \in L_{\phi}^{q}(\partial \Omega)$, $q \geq 2$. There exists a positive constant $\epsilon$ depending on $\Omega$ such that if $q \in[2,2+\epsilon)$, then system (2.1), admits a solution $(\boldsymbol{u}, p) \in\left[W_{\sigma}^{1 / q, q}(\Omega) \cap C^{\infty}(\Omega)\right] \times C^{\infty}(\Omega)$, $\boldsymbol{u} \stackrel{\mathrm{nt}}{\longrightarrow} \boldsymbol{a}$ and

$$
\|\boldsymbol{u}\|_{W^{1 / q, q}(\Omega)} \leq c\|\boldsymbol{a}\|_{L^{q}(\partial \Omega)} .
$$

Moreover:

- (i) if $\boldsymbol{a} \in W^{1, q}(\partial \Omega), q \in[2,2+\epsilon)$, then $\boldsymbol{u} \in W^{1+1 / q, q}(\Omega)$.

- For $n=3$ there are two positive constants $\epsilon$ and $\alpha_{0}$, depending on the Lipschitz character of $\partial \Omega$ such that:

(ii) if $\boldsymbol{a} \in L^{q}(\partial \Omega), q \in[2,+\infty]$, then $\boldsymbol{u} \in W^{1 / q, q}(\Omega)$ and (2.2) holds;

(iii) if $\boldsymbol{a} \in W^{1-1 / q, q}(\partial \Omega), q \in[2,3+\epsilon)$, then

$$
\|\boldsymbol{u}\|_{W^{1, q}(\Omega)} \leq c\|\boldsymbol{a}\|_{W^{1-1 / q, q(\partial \Omega)}} ;
$$

(iv) if $\boldsymbol{a} \in C^{0, \alpha}(\partial \Omega), \alpha \in\left[0, \alpha_{0}\right)$, then

$$
\|\boldsymbol{u}\|_{C^{0, \alpha}(\bar{\Omega})} \leq c\|\boldsymbol{a}\|_{C^{0, \alpha}(\partial \Omega)} .
$$


- If $n=4$ and $\boldsymbol{a} \in L^{3}(\partial \Omega)$, then

$$
\|\boldsymbol{u}\|_{L^{4}(\Omega)} \leq c\|\boldsymbol{a}\|_{L^{3}(\partial \Omega)} .
$$

If $\Omega$ is of class $C^{1}$, then properties (i)-(iv) are satisfied for all $n \geq 2$ with $q \in$ $(1,+\infty), \epsilon=+\infty$ and $\alpha_{0}=1$. In particular, if $n \geq 5$ and $\boldsymbol{a} \in L^{n-1}(\partial \Omega)$, then

$$
\|\boldsymbol{u}\|_{L^{n}(\Omega)} \leq c\|\boldsymbol{a}\|_{L^{n-1}(\partial \Omega)} .
$$

Theorem 2.2. Let $\Omega$ be a bounded domain of $\mathbb{R}^{n}$ of class $C^{2,1}$ and let a $\in$ $W_{\phi}^{-1 / q, q}(\partial \Omega)$, with $q \in(1,+\infty)$. Then system $(2.1)$, admits a solution $(\boldsymbol{u}, p) \in$ $C_{\sigma}^{\infty}(\Omega) \times C^{\infty}(\Omega)$ such that $\boldsymbol{u}$ takes the boundary value $\boldsymbol{a}$ in the sense of the space $W^{-1 / q, q}(\partial \Omega)^{1}$ and

$$
\|\boldsymbol{u}\|_{L^{q}(\Omega)} \leq c\|\boldsymbol{a}\|_{W^{-1 / q, q(\partial \Omega)}} .
$$

Theorem 2.3. Let $\Omega$ be an exterior domain of $\mathbb{R}^{3}$. If $\boldsymbol{a} \in L^{\infty}(\partial \Omega)$, then system (2.1) admits a solution $(\boldsymbol{u}, p) \in\left[L_{\sigma}^{\infty}(\Omega, r) \cap C^{\infty}(\Omega)\right] \times\left[L^{\infty}\left(C S_{R}, r^{2}\right) \cap C^{\infty}(\Omega)\right]$, $\boldsymbol{u} \stackrel{\mathrm{nt}}{\longrightarrow} \boldsymbol{a}$ and

$$
\|\boldsymbol{u}\|_{L^{\infty}(\Omega, r)} \leq c\|\boldsymbol{a}\|_{L^{\infty}(\partial \Omega)} .
$$

Moreover, property (iv) in Theorem 2.1 holds unchanged.

\section{Existence theorems for the Navier-Stokes system}

Thanks to the results just recalled concerning the Stokes problem (2.1), we are in a position to extend the existence results of Fujita-Morimoto [3] to data in $L_{\phi}^{q}(\partial \Omega)$ for Lipschitz domain and in $W_{\phi}^{-1 / q, q}(\partial \Omega)$ for domains of class $C^{2,1}$, for suitable $q$. Moreover, we also prove an existence theorem in Lipschitz exterior domains with data in $L^{\infty}(\partial \Omega)$.

Theorem 3.1. Let $\Omega$ be a Lipschitz, bounded domain of $\mathbb{R}^{3}$ and let

$$
\boldsymbol{a}=\mu \boldsymbol{u}_{0 \mid \partial \Omega}+\boldsymbol{\gamma}
$$

where $\mu \in \mathbb{R}$, $\boldsymbol{u}_{0}=\nabla \beta$, with $\beta \in W^{2, q}(\Omega)(q>3 / 2)$ harmonic function, and $\gamma \in L_{\phi}^{2}(\partial \Omega)$. Then, for every $\mu / v \in \mathbb{R} \backslash G$, with $G$ countable subset of $\mathbb{R}$, there exists a constant $\kappa=\kappa\left(\Omega, v, \boldsymbol{u}_{0}, \mu\right)$ such that, if

$$
\|\boldsymbol{\gamma}\|_{L^{2}(\partial \Omega)} \leq \kappa
$$

then system (1.1)-(1.3) has a solution

$$
(\boldsymbol{u}, p) \in\left[W_{\sigma}^{1 / 2,2}(\Omega) \cap C^{\infty}(\Omega)\right] \times C^{\infty}(\Omega) .
$$

${ }^{1}$ See [10]. 
Proof. Recall that the fundamental solution to the Stokes equation is expressed by

$$
\begin{aligned}
& \mathcal{U}(x-y)=\frac{1}{8 \pi v}\left[\frac{1}{|x-y|}+\frac{(x-y) \otimes(x-y)}{|x-y|^{3}}\right], \\
& \mathcal{P}(x-y)=\frac{1}{4 \pi} \frac{x-y}{|x-y|^{3}},
\end{aligned}
$$

where 1 denotes the unit second-order tensor.

Let $\boldsymbol{u} \in L_{\sigma}^{3}(\Omega)$. By classical results the linear operator

$$
\hat{\mathcal{L}}[\boldsymbol{u}](x)=-\int_{\Omega} \mathcal{U}(x-y)\left[\boldsymbol{u}_{0} \cdot \nabla \boldsymbol{u}+\boldsymbol{u} \cdot \nabla \boldsymbol{u}_{0}\right](y)
$$

maps $L_{\sigma}^{3}(\Omega)$ into $W^{1, t}(\Omega)$ for some $t>3 / 2$. Let $\mathcal{L}_{0}[\boldsymbol{u}]$ be the solution to the Stokes problem with boundary datum $-\operatorname{tr} \hat{\mathcal{L}}[\boldsymbol{u}] \in W^{1-1 / t, t}(\partial \Omega)$. Since by (2.3) and the trace theorem

$$
\left\|\mathcal{L}_{0}[\boldsymbol{u}]\right\|_{W^{1, t}(\Omega)} \leq c\|\operatorname{tr} \hat{\mathcal{L}}[\boldsymbol{u}]\|_{W^{1-1 / t, t}(\partial \Omega)} \leq\|\hat{\mathcal{L}}[\boldsymbol{u}]\|_{W^{1, t}(\Omega)}
$$

we see that also the linear operator $\mathcal{L}_{0}$ maps $L_{\sigma}^{3}(\Omega)$ into $W^{1, t}(\Omega)$. Therefore, by Rellich's compactness theorem, the operator

$$
\mathcal{L}=\hat{\mathcal{L}}+\mathcal{L}_{0}
$$

maps compactly $L_{\sigma}^{3}(\Omega)$ into itself and $\operatorname{tr} \mathcal{L}[\boldsymbol{u}]=\mathbf{0}$ on $\partial \Omega$.

Set

$$
\mathcal{F}=\mathcal{J}-\frac{\mu}{v} \mathcal{L},
$$

where $\mathcal{J}$ denotes the identity map. By classical results there is a countable subset $G$ of $\mathbb{R}$, with a possible accumulation at 0 , such that $\mathcal{F}$ is invertible for all $\mu / \nu \notin G$.

The nonlinear operator

$$
\hat{\mathcal{N}}[\boldsymbol{u}](x)=-\frac{1}{v} \int_{\Omega} \mathcal{U}(x-y)(\boldsymbol{u} \cdot \nabla \boldsymbol{u})(y)
$$

maps $L_{\sigma}^{3}(\Omega)$ into $W_{\sigma}^{1,3 / 2}(\Omega)$ and it holds

$$
\|\hat{\mathcal{N}}[\boldsymbol{u}]\|_{W^{1,3 / 2}(\Omega)} \leq c\|\boldsymbol{u}\|_{L^{3}(\Omega)}^{2} .
$$

Let $\mathcal{N}_{0}[\boldsymbol{u}]$ be the solution to the Stokes problem with boundary datum $-\operatorname{tr} \hat{\mathcal{N}}[\boldsymbol{u}]$. By the trace theorem and (3.2) we have

$$
\left\|\mathcal{N}_{0}[\boldsymbol{u}]\right\|_{L^{3}(\Omega)} \leq c\|\operatorname{tr} \hat{\mathcal{N}}[\boldsymbol{u}]\|_{W^{1 / 3,3 / 2}(\partial \Omega)} \leq\|\hat{\mathcal{N}}[\boldsymbol{u}]\|_{W^{1,3 / 2}(\Omega)} \leq c\|\boldsymbol{u}\|_{L^{3}(\Omega)}^{2} .
$$


Of course, the operator

$$
\mathcal{N}=\hat{\mathcal{N}}+\mathcal{N}_{0}
$$

maps $L_{\sigma}^{3}(\Omega)$ into itself and $\operatorname{tr} \mathcal{N}[\boldsymbol{u}]=\mathbf{0}$ on $\partial \Omega$.

For $\mu / \nu \notin G$ consider the map

$$
\boldsymbol{u}^{\prime}=\stackrel{-1}{\mathcal{F}}\left[\boldsymbol{u}_{\gamma}+\mathcal{N}[\boldsymbol{u}]\right]
$$

from $L_{\sigma}^{3}(\Omega)$ into itself, where $\boldsymbol{u}_{\gamma}$ is the solution to the Stokes problem with boundary datum $\gamma$. Since

$$
\|\stackrel{-1}{\mathcal{F}}[\mathcal{N}[\boldsymbol{u}]]\|_{L^{3}(\Omega)} \leq c_{0}\|\boldsymbol{u}\|_{L^{3}(\Omega)}^{2},
$$

taking into account (2.2), if $\|\boldsymbol{\gamma}\|_{L^{2}(\partial \Omega)}$ is chosen such that

$$
\left\|\stackrel{-1}{\mathcal{F}}\left[\boldsymbol{u}_{\gamma}\right]\right\|_{L^{3}(\Omega)}<\frac{1}{4 c_{0}},
$$

then (3.3) is a contraction in the ball

$$
\mathcal{S}=\left\{\boldsymbol{u} \in L_{\sigma}^{3}(\Omega):\|\boldsymbol{u}\|_{L^{3}(\Omega)} \leq \frac{1}{2 c_{0}}\right\} .
$$

Therefore, by a classical theorem of $\mathrm{S}$. Banach, there is a unique field $\boldsymbol{u} \in \mathcal{S}$ such that

$$
\boldsymbol{u}=\stackrel{-1}{\mathcal{F}}\left[\boldsymbol{u}_{\gamma}+\mathcal{N}[\boldsymbol{u}]\right]
$$

Hence it follows that $\boldsymbol{u}$ is a solution to the equation

$$
\boldsymbol{u}=\boldsymbol{u}_{\gamma}+\frac{\mu}{v} \mathcal{L}[\boldsymbol{u}]+\mathcal{N}[\boldsymbol{u}] .
$$

Since $\boldsymbol{u}_{0}$ is a solution to both Stokes and Navier-Stokes equations, by taking also into account standard regularity theory we see that the field $\mu \boldsymbol{u}_{0}+\boldsymbol{u} \in C^{\infty}(\Omega)$ is a solution to equations (1.1)-(1.2) for a suitable pressure field $p \in C^{\infty}(\Omega)$. This solution assumes the boundary datum in the sense that $\boldsymbol{u}_{0} \stackrel{\mathrm{nt}}{\longrightarrow} \boldsymbol{u}_{0 \mid \partial \Omega}, \boldsymbol{u}_{\gamma} \stackrel{\mathrm{nt}}{\longrightarrow} \boldsymbol{\gamma}$ and $\mathcal{N}[\boldsymbol{u}], \mathcal{L}[\boldsymbol{u}]$ have zero trace on $\partial \Omega$ as elements of the Sobolev space $W_{0}^{1,3 / 2}(\Omega)$ (see also Remark 3.2).

Remark 3.2. Assume for simplicity that $\beta$ is a regular harmonic function. By the regularity results for the Stokes problem we have, in particular, that if the norm of $\gamma$ is small in the corresponding function space, then

- if $\boldsymbol{\gamma} \in L^{q}(\partial \Omega), q>4$, and $\boldsymbol{u}_{0} \in W^{1, t}(\Omega), t>3$, then $\boldsymbol{u} \stackrel{\mathrm{nt}}{\longrightarrow} \boldsymbol{a}$;

- if $\boldsymbol{\gamma} \in L^{\infty}(\partial \Omega)$, then $\boldsymbol{u} \in L^{\infty}(\Omega)$ 
and there are two positive constants $\epsilon$ and $\alpha_{0}(<1)$ depending on $\Omega$ such that

- if $\boldsymbol{\gamma} \in L^{s}(\partial \Omega), s \in[2,2+\epsilon)$, then $\boldsymbol{u} \in W^{1 / s, s}(\Omega)$,

- if $\boldsymbol{\gamma} \in W^{1-1 / s, s}(\partial \Omega), s \in[3 / 2,3+\epsilon)$, then $\boldsymbol{u} \in W^{1, s}(\Omega)$,

- if $\boldsymbol{\gamma} \in C^{0, \alpha}(\partial \Omega), \alpha \in\left[0, \alpha_{0}\right)$, then $\boldsymbol{u} \in C^{0, \alpha}(\bar{\Omega})$.

If $\Omega$ is of class $C^{1}$, then the above constants $\epsilon$ and $\alpha_{0}$ can be taken arbitrarily large and equal to 1 respectively. Standard regularity results also hold for the pressure field $p$.

Remark 3.3. In virtue of Theorem 2.1 and estimates (2.3), (2.4), (2.5), existence theorems like the above one can also be established for all $n \geq 2$. If $n=2$ we can take $\boldsymbol{a} \in L^{2-\epsilon}(\partial \Omega)$ with $\epsilon$ depending on $\Omega\left(\boldsymbol{a} \in L^{q}(\partial \Omega), q>1\right.$, for $\Omega$ of class $C^{1}$ ); if $n=4$, we have to require $a \in L^{3}(\partial \Omega)$; if $n>4, \Omega$ must be of class $C^{1}$ and $\boldsymbol{a} \in L^{n-1}(\partial \Omega)$.

Taking into account Theorem 2.2 and estimate (2.2), following the argument in the proof of the above Theorem it is not difficult to get:

Theorem 3.4. Let $\Omega$ be a bounded domain of $\mathbb{R}^{n}$ of class $C^{2,1}$ and let a be given by (3.1) with $\mu \in \mathbb{R}, \boldsymbol{u}_{0}=\nabla \beta, \beta \in W^{2, q}(\Omega)(q>n)$ harmonic function and $\gamma \in W_{\phi}^{-1 / n, n}(\partial \Omega)$. There is a countable subset $G \subset \mathbb{R}$ such that, for $\mu / \nu \notin G$, there is a constant $\kappa=\kappa\left(\Omega, v, \boldsymbol{u}_{0}, \mu\right)$ such that, if

$$
\|\boldsymbol{\gamma}\|_{W^{-1 / n, n}(\partial \Omega)} \leq \kappa
$$

then (1.1)-(1.3) has a solution

$$
(\boldsymbol{u}, p) \in\left[L_{\sigma}^{n}(\Omega) \cap C^{\infty}(\Omega)\right] \times C^{\infty}(\Omega) .
$$

Taking into account the result of $\mathrm{H}$. Morimoto recalled in the introduction, we also have:

Theorem 3.5. Let $\Omega$ be the annulus

$$
\Omega=\left\{x \in \mathbb{R}^{2}: R_{1}<|x|<R_{2}\right\},
$$

and let $\boldsymbol{a}$ be given by (3.1), with $\boldsymbol{u}_{0} \in W^{1, q}(\Omega), q>1, \boldsymbol{\gamma} \in L_{\phi}^{q}(\Omega)$. If $\|\boldsymbol{\gamma}\|_{L^{q}(\partial \Omega)}$ is sufficiently small, then (1.1)-(1.3) has a solution

$$
(\boldsymbol{u}, p) \in\left[W_{\sigma}^{1 / q, q}(\Omega) \cap C^{\infty}(\Omega)\right] \times C^{\infty}(\Omega) .
$$

Let us pass to treat the case of an exterior domain. The following theorem holds.

Theorem 3.6. Let $\Omega$ be an exterior Lipschitz domain of $\mathbb{R}^{3}$ and let $\boldsymbol{a}$ be expressed by (3.1) where $\boldsymbol{u}_{0 \mid \partial \Omega}, \boldsymbol{\gamma} \in L^{\infty}(\partial \Omega)$ and $\boldsymbol{u}_{0}=O\left(r^{-2}\right)$. There is a countable subset $G \subset \mathbb{R}$ such that, for $\mu / \nu \notin G$, there is a constant $\xi=\xi\left(\Omega, \nu, \boldsymbol{u}_{0}, \mu\right)$ such that, if $\|\boldsymbol{\gamma}\|_{L^{\infty}(\partial \Omega)} \leq \xi$, then the Navier-Stokes problem admits a solution

$$
(\boldsymbol{u}, p) \in\left[L_{\sigma}^{\infty}(\Omega, r) \cap C^{\infty}(\Omega)\right] \times\left[L^{\infty}\left(\complement S_{R}, r^{2} \log r\right) \cap C^{\infty}(\Omega)\right]
$$

and $\boldsymbol{u} \stackrel{\mathrm{nt}}{\longrightarrow} \boldsymbol{a}$. 
Proof. By well-known results about the behavior at infinity of volume potential (see, e.g., [6] Lemma II.7.2), the operator

$$
\hat{\mathcal{V}}[\boldsymbol{u}](x)=-\nabla \int_{\Omega} \mathcal{U}(x-y)\left(\boldsymbol{u} \otimes \boldsymbol{u}_{0}+\boldsymbol{u}_{0} \otimes \boldsymbol{u}\right)(y)
$$

maps boundedly $L^{\infty}(\Omega, r)$ into $L^{\infty}\left(\Omega, r^{2-\eta}\right)$ for every $\eta \in(0,1)$. Hence $\hat{\mathcal{V}}[\boldsymbol{u}] \in$ $L^{q}(\Omega)$, for every $q>3 / 2$. Moreover, by Calderón-Zygmund's theorem $\nabla \hat{\mathcal{V}}$ maps boundedly $L^{\infty}(\Omega, r)$ into $L^{q}(\Omega)$, for every $q>3 / 2$.

Let $\left\{\boldsymbol{u}_{k}\right\}_{k \in \mathbb{N}}$ be a bounded sequence in $L_{\sigma}^{\infty}(\Omega, r)$. By what we said above $\hat{v}\left[\boldsymbol{u}_{k}\right]$ is bounded in $W^{1, q}(\Omega)$ for every $q>3 / 2$ so that we can extract from it a subsequence, we denote by the same symbol, which converges uniformly to a field $\boldsymbol{u}$ in $C_{\mathrm{loc}}(\bar{\Omega})$. On the other hand

$$
\begin{aligned}
\left\|\hat{\mathcal{V}}\left[\boldsymbol{u}_{k}-\boldsymbol{u}_{h}\right]\right\|_{L^{\infty}(\Omega, r)} & \\
& \leq R\left\|\hat{\mathcal{V}}\left[\boldsymbol{u}_{k}-\boldsymbol{u}_{h}\right]\right\|_{C\left(\bar{\Omega} \cap S_{R}\right)}+R^{\eta-1}\left\|r^{2-\eta \hat{V}}\left[\boldsymbol{u}_{k}-\boldsymbol{u}_{h}\right]\right\|_{L^{\infty}\left(C S_{R}\right)} \\
& \leq R\left\|\hat{\mathcal{V}}\left[\boldsymbol{u}_{k}-\boldsymbol{u}_{h}\right]\right\|_{C\left(\bar{\Omega} \cap S_{R}\right)}+c R^{\eta-1} .
\end{aligned}
$$

Let $\epsilon>0$ and let $m$ be such that for every $h, k>m,\left\|\hat{\mathcal{V}}\left[\boldsymbol{u}_{k}-\boldsymbol{u}_{h}\right]\right\|_{C\left(\bar{\Omega} \cap S_{R}\right)}<$ $\epsilon /(2 R)$, with $R^{1-\eta}>2 c / \epsilon$. Therefore, from the above relation it follows that $\left\|\hat{\mathcal{V}}\left[\boldsymbol{u}_{k}-\boldsymbol{u}_{h}\right]\right\|_{L^{\infty}(\Omega, r)}<\epsilon$ for all $h, k>m$ so that $\hat{\mathcal{V}}\left[\boldsymbol{u}_{k}\right]$ is a Cauchy sequence in $L_{\sigma}^{\infty}(\Omega, r)$ and the operator $\hat{V}$ is compact from $L_{\sigma}^{\infty}(\Omega, r)$ into itself. Let $\mathcal{V}_{0}[\boldsymbol{u}]$ be the solution to the Stokes problem with boundary datum $-\operatorname{tr} \hat{\mathcal{V}}[\boldsymbol{u}]$. It is not difficult to see that $\mathcal{V}_{0}$ maps compactly $L^{\infty}(\Omega, r)$ into itself. Set

$$
\mathcal{G}=\mathcal{J}-\frac{\mu}{v} \nu
$$

with $\mathcal{V}=\hat{\mathcal{V}}+\mathcal{V}_{0}$. Since $\mathcal{G}$ is compact, there is a countable subset $G \subset \mathbb{R}$ such that $\mathcal{G}$ is invertible for all $\mu / \nu \notin G$.

The operators

$$
\hat{\mathcal{W}}[\boldsymbol{u}]=-\frac{1}{v} \nabla \int_{\Omega} \mathcal{U}(x-y)(\boldsymbol{u} \otimes \boldsymbol{u})(y)
$$

and $\mathcal{W}_{0}[\boldsymbol{u}]$, solution to the Stokes problem with datum $-\operatorname{tr} \hat{\mathcal{W}}[\boldsymbol{u}]$, map $L^{\infty}(\Omega, r)$ into itself. Consider the map

$$
\boldsymbol{u}^{\prime}=\stackrel{-1}{\mathcal{G}}\left[\boldsymbol{u}_{\gamma}+\mathcal{W}[\boldsymbol{u}]\right]
$$

for $\mu \notin G$, where $\mathcal{W}=\hat{\mathcal{W}}+\mathcal{W}_{0}$ and $\boldsymbol{u}_{\gamma}$ is the solution to the Stokes problem with boundary datum $\gamma$. It is not difficult to see that

$$
\|\stackrel{-}{\mathcal{G}}[\mathcal{W}[\boldsymbol{u}]]\|_{L^{\infty}(\Omega, r)} \leq c_{0}^{\prime}\|\boldsymbol{u}\|_{L^{\infty}(\Omega, r)}^{2} .
$$


Therefore, taking into account (2.7), if $\|\boldsymbol{\gamma}\|_{L^{\infty}(\partial \Omega)}$ is chosen such that

$$
\left\|\stackrel{-1}{\mathcal{G}}\left[\boldsymbol{u}_{\gamma}\right]\right\|_{L^{3}(\Omega)}<\frac{1}{4 c_{0}^{\prime}},
$$

then the map (3.4) has a fixed point $\boldsymbol{u}$ and the field $\boldsymbol{u}+\mu \boldsymbol{u}_{0}$ is a solution to the Navier-Stokes problem.

Remark 3.7. Of course, if $\boldsymbol{u}_{0}$ and $\boldsymbol{\gamma}$ are more regular, then so does the solution $(\boldsymbol{u}, p)$. In particular if $\boldsymbol{u}_{0}, \boldsymbol{\gamma} \in C(\partial \Omega)$, then is $\boldsymbol{u} \in C(\bar{\Omega})$.

Remark 3.8. In virtue of the result in [13] the derivatives of $\boldsymbol{u}$ have the following behavior at infinity

$$
\underbrace{\nabla \ldots \nabla}_{k \text { times }} \boldsymbol{u}=O\left(r^{-1-k}\right)
$$

and

$$
p=O\left(r^{-2} \log r\right), \quad \underbrace{\nabla \ldots \nabla}_{k \text { times }} p=O\left(r^{-2-k}\right),
$$

with $k \in \mathbb{N}$.

Remark 3.9. As far as uniqueness of the solutions in the above theorems are concerned, we quote [10], and [6, Chapter IX]. The existence of a solution to system (1.1)-(1.3) in a Lipschitz exterior domain, with $\boldsymbol{a} \in L^{\infty}(\partial \Omega)$, which converges at infinity to an assigned nonzero constant vector has been recently proved in [11].

\section{References}

[1] W. BorChers and K. PILECKAS, Note on the flux problem for stationary incompressible Navier-Stokes equations in domains with a multiply connected boundary, Acta Appl. Math. 37 (1994), 21-30.

[2] R.M. BROWN and Z. SHEN, Estimates for the Stokes operator in Lipschitz domains, Indiana Univ. Math. J. 44 (1995), 1183-1206.

[3] H. Fujita and H. Morimoto, A remark on the existence of the Navier-Stokes flow with non-vanishing outflow condition, GAKUTO Internat. Ser. Math. Sci. Appl. 10 (1997), 5361 ,

[4] H. Fujita, H. Morimoto and H. Окамото, Stability analysis of Navier-Stokes flows in annuli, Math. Methods Appl. Sci. 20 (1997), 959-978,

[5] G. P. GALDI, On the existence of steady motions of a viscous flow with nonhomogeneous boundary conditions, Matematiche (Catania) 46 (1991), 503-524.

[6] G. P. GALDI, "An Introduction to the Mathematical Theory of the Navier-Stokes Equations", Vol. I, II, revised edition, Springer Tracts in Natural Philosophy, C. Truesdell (ed.), Vol. 38, Springer-Verlag, 1998.

[7] M. Mitrea and M. TAYLOR, Navier-Stokes equations on Lipschitz domains in Riemannian manifolds, Math. Ann. 321 (2001), 955-987.

[8] H. Morimoto, Note on the boundary value problem for the Navier-Stokes equations in 2$D$ domain with general outflow condition (in Japanese), Memoirs of the Institute of Science and Technology, Meiji University 35 (1997), 95-102. 
[9] H. Morimoto, General outflow condition for Navier-Stokes system, In: "Recent Topics on Mathematical Theory of Viscous Incompressible fluid", Lectures Notes in Num. Appl. Anal., Vol. 16, 1998, 209-224.

[10] R. Russo, On the existence of solutions to the stationary Navier-Stokes equations, Ricerche Mat. 52 (2003), 285-348.

[11] R. RUSSO and C. G. SIMADER, A note on the existence of solutions to the Oseen system in Lipschitz domains, J. Math. Fluid Mech. 8 (2006), 64-76.

[12] Z. SHEN, A note on the Dirichlet problem for the Stokes system in Lipschitz domains, Proc. Amer. Math. Soc. 123 (1995), 801-811.

[13] V. SVERÁK and T-P TSAI, On the spatial decay of 3-D steady-state Navier-Stokes flows, Comm. Partial Differential Equations 25 (2000), 2107-2117.

Dipartimento di Matematica

Seconda Università degli Studi di Napoli Via Vivaldi, 43

I-81100 Caserta, Italy

a.russo@unina2.it

giulio.starita@unina2.it 